\title{
Sex differences in delayed cerebral ischemia after subarachnoid hemorrhage
}

\author{
Menno R. Germans, MD, PhD, ${ }^{1}$ Blessing N. R. Jaja, MD, PhD, ${ }^{2}$ \\ Airton Leonardo de Oliviera Manoel, MD, ${ }^{2}$ Ashley H. Cohen, $\mathrm{MSc},{ }^{3}$ and \\ R. Loch Macdonald, MD, PhD, FRCS(C), ${ }^{4,5}$ on behalf of the SAHIT collaborators
}

\begin{abstract}
${ }^{1}$ Department of Neurosurgery, Radboud University Medical Center, Nijmegen, The Netherlands; ${ }^{2}$ Neuroscience Research Program, ${ }^{3}$ Applied Health Centre, and ${ }^{4}$ Division of Neurosurgery, Department of Surgery, Li Ka Shing Knowledge Institute, St. Michael's Hospital; and ${ }^{5}$ Faculty of Medicine, University of Toronto, Ontario, Canada
\end{abstract}

OBJECTIVE In this study the authors sought to investigate the sex differences in the risk of delayed cerebral ischemia $(\mathrm{DCl})$, delayed cerebral infarction, and the role of hormonal status.

METHODS Ten studies included in the SAHIT (SAH International Trialists) repository were analyzed using a fitting logistic regression model. Heterogeneity between the studies was tested using $\mathrm{I}^{2}$ statistics, and the results were pooled using a random-effects model. Multivariable analysis was adjusted for the effects of neurological status and fixed effect of study. An additional model was examined in which women and men were split into groups according to an age cut point of 55 years, as a surrogate to define hormonal status.

RESULTS A pooled cohort of 6713 patients was analyzed. The risk of $\mathrm{DCl}$ was statistically significantly higher in women than in men (OR 1.29, 95\% Cl 1.12-1.48); no difference was found with respect to cerebral infarction (OR 1.17, 95\% Cl $0.98-1.40)$. No difference was found in the risk of $\mathrm{DCl}$ when comparing women $\leq 55$ and $>55$ years $(\mathrm{OR} 0.87,95 \% \mathrm{Cl}$ $0.74-1.02 ; p=0.08$ ) or when comparing men $\leq 55$ and $>55$ years $(p=0.38)$. Independent predictors of $\mathrm{DCl}$ were World Federation of Neurosurgical Societies (WFNS) grade, Fisher grade, age, and sex. Independent predictors of infarction included WFNS grade, Fisher grade, and aneurysm size.

CONCLUSIONS Female sex is associated with a higher risk of $\mathrm{DCl}$. Sex differences may play a role in the pathogenesis of $\mathrm{DCl}$ but are not associated with menopausal status. The predictors of $\mathrm{DCl}$ and cerebral infarction were identified in a very large cohort and reflect experience from multiple institutions.

https://thejns.org/doi/abs/10.3171/2017.3.JNS162808

KEY WORDS brain ischemia; delayed cerebral ischemia; sex; subarachnoid hemorrhage; cohort study; risk factor in epidemiology; vascular disorders

$\Lambda$ PPROXIMATELY $30 \%$ of patients with ruptured intracranial aneurysms causing subarachnoid hemorrhage $(\mathrm{SAH})$ develop delayed cerebral ischemia (DCI) or delayed cerebral infarction. ${ }^{6,21,24}$ These complications of SAH are recognized as the most important reasons for poor outcome; hence, considerable effort is devoted to monitoring and preventing their occurrence or mitigating their effect during hospital admission. , $^{61,24}$ There is a growing realization that DCI and delayed cerebral infarction have a complex pathophysiology involving the interaction of multiple mechanisms that are the subject of intense research. ${ }^{2,16}$ Women have a disproportionately higher incidence of intracranial aneurysms than men, particularly after the menopausal period, an indication that sex-specific factors may mediate, in part, the formation of intracranial aneurysms, and likely by extension the pathogenesis of SAH.

Clinical studies have identified a number of predictors of DCI and cerebral infarction. ${ }^{3}$ There are limited data regarding the effect of sex, and the available evidence has been contradictory. In this study, we investigated the relationship of sex to DCI and delayed cerebral infarction. The study may help improve knowledge about the pathophysiology of DCI and delayed cerebral infarction, or contribute

ABBREVIATIONS DCI = delayed cerebral ischemia; IQR = interquartile range; $\mathrm{SAH}=$ subarachnoid hemorrhage; SAHIT = Subarachnoid Hemorrhage International Trialists; WFNS $=$ World Federation of Neurosurgical Societies.

SUBMITTED November 8, 2016. ACCEPTED March 17, 2017.

INCLUDE WHEN CITING Published online September 1, 2017; DOI: 10.3171/2017.3.JNS162808. 
to better risk assessment for delayed complications after SAH.

\section{Methods \\ Study Population}

After receiving approval from the institutional review board of St. Michael's Hospital, Toronto, we selected studies from the Subarachnoid Hemorrhage International Trialists (SAHIT) data repository that used nimodipine as a standard treatment and reported DCI ("delayed cerebral ischemia" or "therapeutic hypertension" or "delayed ischemic neurological deficit") or cerebral infarction ("cerebral infarction"). The repository contains data of multiple clinical trials and observational prospective databases regarding aneurysmal SAH. Ten data sets were selected, including those of the British Aneurysm Nimodipine trial (BRANT); Clazosentan to Overcome Neurological Ischemia and Infarction Occurring After SAH (CONSCIOUS-1); University of Washington Database of Subarachnoid Treatment (D-SAT); Acute systemic erythropoietin therapy to reduce delayed ischemic deficits following aneurysmal subarachnoid hemorrhage and effects of acute treatment with statins on cerebral autoregulation in patients after aneurysmal subarachnoid hemorrhage trials (EPO_STATIN); Heinrich Heine University Concomitant Intraventricular Fibrinolysis and Low-Frequency Rotation After Severe Subarachnoid Hemorrhage Trial (HHU); Intraoperative Hypothermia for Aneurysm Surgery Trial (IHAST); Intravenous Magnesium Sulfate for Aneurysmal Subarachnoid Hemorrhage (IMASH) trial; International Subarachnoid Aneurysm Trial (ISAT); Kurashiki Central Hospital, Japan; and Columbia University Subarachnoid Hemorrhage Outcomes Project (SHOP). The objective and structure of the SAHIT database has been reported previously. ${ }^{12,17}$

\section{Study Parameters}

Based on the current knowledge about factors predicting DCI and cerebral infarction, the following variables were selected for analysis: sex, age, neurological status at admission (World Federation of Neurosurgical Societies [WFNS] grade $^{23}$ ), amount of subarachnoid blood seen on the initial CT scan (Fisher grade ${ }^{7}$ ), smoking, diabetes mellitus, hyperglycemia, hydrocephalus, history of hypertension, location and size of aneurysm, and treatment modality (coiling or clipping)..$^{3-5,8,14,16,20,25}$ To study the effect of menopausal status on DCI and cerebral infarction, we created a dummy variable, "status," as a surrogate for menopausal status using age cutoff $\leq 55$ years or $>55$ years. This age cut point was based on previous studies. ${ }^{1,19}$ Therefore, 4 groups were analyzed: men $\leq 55$ years, men $>55$ years, women $\leq 55$ years, and women $>55$ years. The primary outcome of interest was DCI, and the secondary outcome was delayed cerebral infarction, as defined by each individual data set (Supplemental Table).

\section{Statistical Analysis}

First, the data were examined descriptively, with categorical variables expressed as counts with percentages and continuous variables described as means with standard deviations. Binary logistic regression models were fitted to obtain the unadjusted odds ratios associated with the effect of sex in each study, and the resultant odds ratios were then pooled using a random-effects model. Betweenstudy heterogeneity was assessed using the $\mathrm{I}^{2}$ statistic. Where the pooled unadjusted odds ratios were statistically significant, further adjusted analysis was performed to account for the effects of age, admission WFNS grade, and the fixed effect of study. We also performed multivariable logistic regression analysis to identify independent predictors of DCI and infarction. Statistical significance was set at $p<0.05$. The analysis was performed using Stata software (version 13.1, StataCorp).

\section{Results}

A pooled cohort of 6713 patients was analyzed, 4406 $(65 \%)$ of whom were women. The study cohort characteristics are shown in Table 1. Women were on average older and had a higher incidence of a premorbid history of hypertension. The other variables were comparably distrib-

TABLE 1. Baseline characteristics of patients in the pooled data set

\begin{tabular}{|c|c|c|}
\hline Variable & Men & Women \\
\hline No. of patients & 2306 & 4406 \\
\hline Mean age in yrs $\pm S D$ & $50.8 \pm 12.9$ & $55.2 \pm 13.8$ \\
\hline Hypertension & $549(33)$ & $1407(39)$ \\
\hline \multicolumn{3}{|l|}{ WFNS grade } \\
\hline I & $1279(50)$ & $2143(45)$ \\
\hline II & $633(25)$ & $1317(27)$ \\
\hline III & $175(7)$ & $330(7)$ \\
\hline IV & $266(10)$ & $572(12)$ \\
\hline V & $231(9)$ & $453(9)$ \\
\hline \multicolumn{3}{|l|}{ Fisher grade } \\
\hline 1 & $185(8)$ & $275(6)$ \\
\hline 2 & $474(21)$ & $908(21)$ \\
\hline 3 & $1061(46)$ & $2130(49)$ \\
\hline 4 & $589(26)$ & $1008(23)$ \\
\hline \multicolumn{3}{|l|}{ Aneurysm location } \\
\hline ACA & $1131(53)$ & $1439(34)$ \\
\hline ICA & $420(20)$ & $1437(34)$ \\
\hline MCA & $408(19)$ & $863(21)$ \\
\hline$P C$ & $180(8)$ & $451(11)$ \\
\hline \multicolumn{3}{|l|}{ Aneurysm diameter in $\mathrm{mm}$} \\
\hline $0-12$ & $1794(83)$ & $3662(86)$ \\
\hline $13-24$ & $199(9)$ & $350(8)$ \\
\hline$\geq 25$ & $157(7)$ & $235(6)$ \\
\hline \multicolumn{3}{|l|}{ Aneurysm treatment } \\
\hline Clip & $1462(67)$ & $2943(69)$ \\
\hline Coil & $731(33)$ & $1333(31)$ \\
\hline $\mathrm{DCl}$ & $375(16)$ & $879(20)$ \\
\hline Delayed cerebral infarct & $236(14)$ & $562(16)$ \\
\hline
\end{tabular}

$\mathrm{ACA}=$ anterior cerebral artery; $\mathrm{ICA}=$ internal carotid artery; $\mathrm{MCA}=$ middle cerebral artery; $\mathrm{PC}=$ posterior circulation.

The table does not include missing data. Values are presented as the number of patients (\%) unless stated otherwise. 
uted between sexes. The incidence of DCI was statistically higher in women than in men (20\% vs $16 \%, \mathrm{p}=0.001)$; similarly, the incidence of infarction was higher in women than in men $(16 \%$ vs $14 \%, \mathrm{p}=0.001)$. A meta-analysis of the pooled unadjusted odds ratios (Fig. 1) indicated that women had a significantly higher risk of DCI in the pooled cohort (OR 1.32, 95\% CI 1.15-1.52), and also a higher risk of infarction (OR 1.24, 95\% CI 1.01-1.52), than men. In a model adjusting for age, WFNS grade, and fixed effect of study, the risk difference remained statistically significant for DCI (OR 1.29, 95\% CI 1.12-1.48), but not for infarction (OR 1.17, 95\% CI 0.98-1.40). The between-study heterogeneity was statistically not significant $\left(\mathrm{I}^{2}\right.$ : $\mathrm{DCI}=0.0 \%, \mathrm{p}$ $=0.53$; infarct $=14.8 \%, \mathrm{p}=0.31$ ). Table 2 shows the analysis of the effect of menopausal status on DCI and infarct. The median age of women who were $\leq 55$ years was 45 years (interquartile range [IQR] 39-50 years); those $>55$ years had a median age of 65 years (IQR 59-72 years). We found no difference in the risk of DCI when comparing women $\leq 55$ years to their counterparts who were $>55$ years (OR $0.87,95 \%$ CI $0.74-1.02 ; \mathrm{p}=0.08$ ). The risk of DCI was significantly lower in men who were $\leq 55$ years and in those $>55$ years, in comparison with women $\leq 55$ years. No difference in DCI risk was found on comparing men $\leq 55$ years to those $>55$ years $(p=0.38)$. We did a sensitivity analysis using the age cut point of 50 years and found no difference in the risk of DCI between women who were younger than 50 years compared with those who were older than 50 years $(\mathrm{p}=0.41)$. The risk of infarction did not differ statistically between the sexes when analyzed based on an age cut point of 55 years.

Table 3 presents results from the multivariable model after including all predictor variables in Table 1. The variables that were independently associated with DCI were WFNS grade $(p<0.001)$, Fisher grade $(p<0.001)$, patient age $(p=0.025)$, and sex $(p=0.007)$. Independent predictors of infarction included WFNS grade, Fisher grade, and aneurysm size.

\section{Discussion}

This study demonstrated a significantly higher risk of DCI in women than in men. Our finding is in consonance with the results of an aggregate-data meta-analysis by De Rooij et al. to identify predictors of DCI following aneurysmal SAH. ${ }^{3}$ The risk difference of $29 \%$ in our study is comparable with the $30 \%$ estimated in their meta-analysis (OR 1.3, 95\% CI 1.1-1.6). Taken together, the available evidence suggests that sex plays some role in the pathogenesis of DCI, although we are uncertain as to which factors mediate this effect. In our present study, the women were older and presented in poorer clinical condition compared with their male counterparts; however, these factors had only marginal impact on the magnitude of the effect of sex

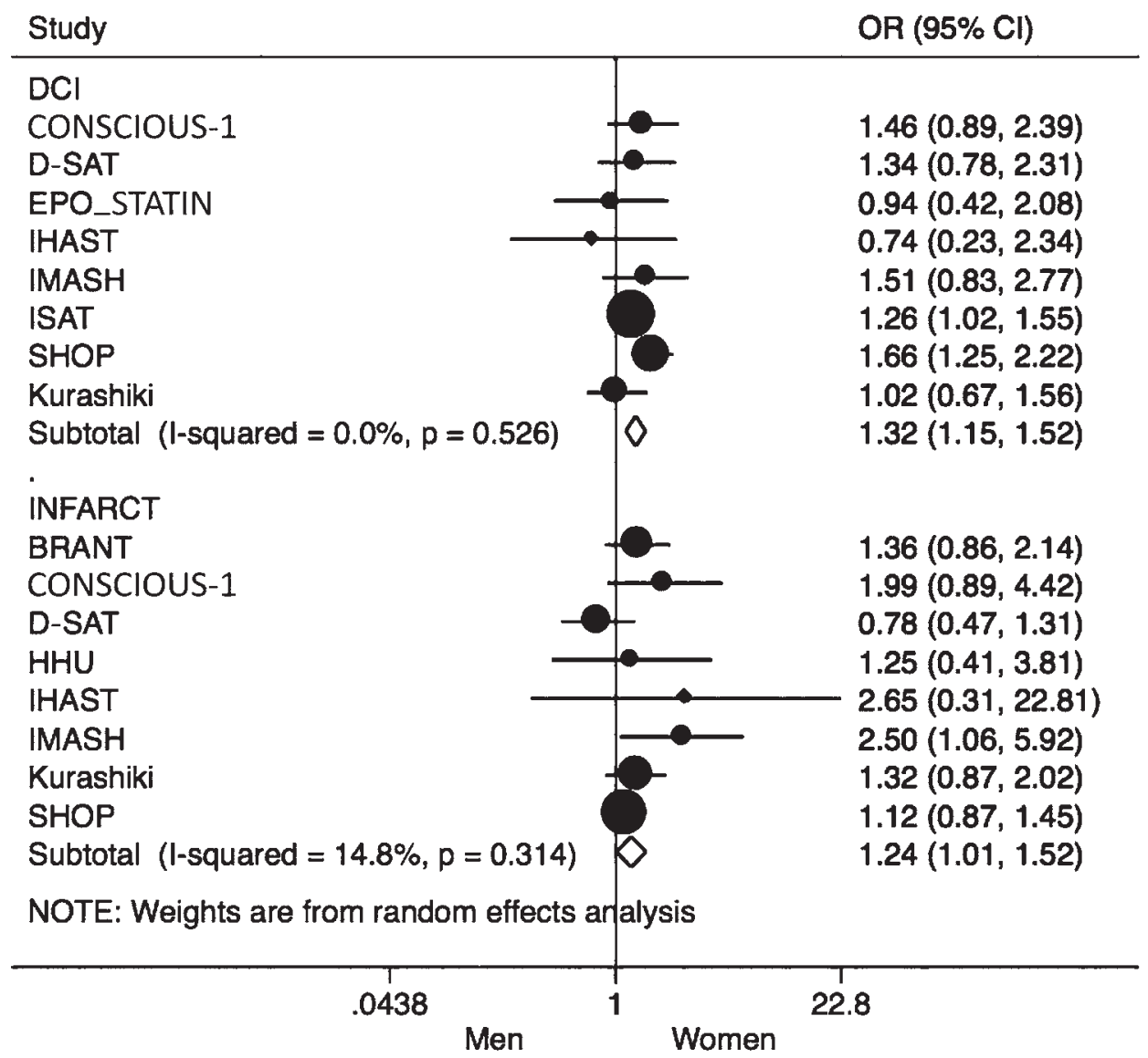

FIG. 1. Forest plot of unadjusted odds ratios showing the effect of sex on DCl and infarcts in the different studies. See Methods for explanation of included studies. 
TABLE 2. Effect of hormonal status on $\mathrm{DCl}$ and infarcts

\begin{tabular}{|c|c|c|c|c|c|}
\hline Variable & Median Age in Yrs (IQR) & $\mathrm{DCl}{ }^{*}$ & $p$ Value & Infarctions* & p Value \\
\hline \multicolumn{6}{|l|}{ Women } \\
\hline$\leq 55 \mathrm{yrs}$ & $45(39-50)$ & Reference & & Reference & \\
\hline$>55 \mathrm{yrs}$ & $65(59-72)$ & $0.87(0.74-1.02)$ & 0.08 & $1.01(0.82-1.24)$ & 0.93 \\
\hline \multicolumn{6}{|l|}{ Men } \\
\hline$\leq 55 \mathrm{yrs}$ & $44(37-50)$ & $0.75(0.62-0.90)$ & 0.002 & $0.81(0.64-1.03)$ & 0.09 \\
\hline$>55 \mathrm{yrs}$ & $61(57-68)$ & $0.72(0.59-0.90)$ & 0.003 & $0.96(0.73-1.25)$ & 0.76 \\
\hline
\end{tabular}

* Data are presented as OR $(95 \% \mathrm{Cl})$.

on DCI, suggesting little or no role for the primary brain injury in mediating the observed risk difference between men and women. Previous studies have reported sex-related differences in aneurysm morphology that could provide a mechanistic explanation for the higher likelihood of DCI in women than in men. ${ }^{9}$ It has been shown that women tend to have a higher prevalence of internal carotid artery aneurysms and that men tend to have a higher prevalence of anterior cerebral artery aneurysms, including anterior communicating artery aneurysms. Lindekleiv et al., using computational fluid dynamic simulation, showed that women have much narrower vessel diameters and higher blood flow velocity that results in higher wall shear stress in the middle cerebral artery and internal carotid artery bifurcations. ${ }^{15}$ Higher wall stress could induce endothelial injury and altered vascular reactivity leading to perfusion deficits and the development of ischemia.

Furthermore, we investigated a putative role for female hormonal status, considering evidence from prior studies suggesting that sex and age interact to influence the risk of SAH, probably acting through a sex-specific hormonal factor. ${ }^{22}$ Menopause typically occurs between the ages of 50 and 59 years when estrogen levels begin to decrease. The higher prevalence of ruptured aneurysms in women compared with men is more pronounced in the postmenopausal period, probably because of loss of the protective

TABLE 3. Multivariable analysis to identify independent predictors of $\mathrm{DCl}$ and infarcts

\begin{tabular}{|c|c|c|c|c|}
\hline Variable & $\mathrm{DCl}^{*}$ & p Value & Infarction* & $\mathrm{p}$ Value \\
\hline Age & $1.00(0.98-1.01)$ & 0.025 & $1.00(0.99-1.01)$ & 0.662 \\
\hline Female sex & $1.38(1.09-1.74)$ & 0.007 & $1.02(0.81-1.30)$ & 0.842 \\
\hline Hypertension & $1.06(0.86-1.31)$ & 0.5803 & $0.91(0.72-1.13)$ & 0.377 \\
\hline WFNS grade & & $<0.001$ & & $<0.001$ \\
\hline 1 & Reference & & Reference & \\
\hline II & $1.87(1.40-2.49)$ & & $1.74(1.27-2.38)$ & \\
\hline III & $3.05(1.89-4.93)$ & & $3.05(1.82-5.13)$ & \\
\hline IV & $3.03(2.28-4.02)$ & & $3.28(2.42-4.44)$ & \\
\hline V & $2.04(1.44-2.88)$ & & $3.84(2.73-5.41)$ & \\
\hline Fisher grade & & $<0.001$ & & 0.003 \\
\hline 1 & Reference & & Reference & \\
\hline 2 & $0.89(0.53-1.50)$ & & $2.38(1.37-4.15)$ & \\
\hline 3 & $1.97(1.22-3.18)$ & & $2.48(1.44-4.26)$ & \\
\hline 4 & $1.51(0.89-2.54)$ & & $2.94(1.66-5.22)$ & \\
\hline Aneurysm location & & 0.07 & & 0.059 \\
\hline $\mathrm{ACA}$ & Reference & & Reference & \\
\hline ICA & $0.79(0.61-1.01)$ & & $1.22(0.93-1.59)$ & \\
\hline MCA & $0.82(0.62-1.08)$ & & $0.79(0.58-1.07)$ & \\
\hline PC & $0.67(0.48-0.94)$ & & $1.11(0.79-1.54)$ & \\
\hline Aneurysm diameter (mm) & & 0.466 & & 0.003 \\
\hline $0-12$ & Reference & & Reference & \\
\hline $13-24$ & $1.23(0.88-1.72)$ & & $1.59(1.14-2.21)$ & \\
\hline$\geq 25$ & $0.98(0.58-1.68)$ & & $1.79(1.10-2.92)$ & \\
\hline Aneurysm treatment & & 0.303 & & 0.354 \\
\hline Clip & Reference & & Reference & \\
\hline Coil & $0.87(0.67-1.14)$ & & $0.88(0.66-1.16)$ & \\
\hline
\end{tabular}

\footnotetext{
${ }^{*}$ Values are presented as OR $(95 \% \mathrm{Cl})$
} 
effect of estrogen on vascular endothelium and smooth muscle integrity during this period. Estrogen regulates a number of inflammatory cascades and contributes to vascular wall integrity. ${ }^{22}$ The dysregulation of these mechanisms has been implicated in the pathogenesis of DCI. ${ }^{16,22}$ We did not find an association between menopausal status and DCI risk in the present study. It is possible that the arbitrary age cut point we used was a crude and insensitive marker of menopausal status. A better approach would have been to directly measure estrogen levels. Furthermore, some studies have suggested that the mechanism by which estrogen influences aneurysm pathogenesis may be related to the fluctuation rather than the absolute deficiency in estrogen levels. ${ }^{22}$

Although a significant relation of sex to cerebral infarction was seen in the pooled odds ratios, the statistical significance was lost after adjusting for other covariates in the analysis. This is in contrast to the relationship between sex and DCI, which remained significant in the multivariable analysis. Multiple mechanisms have been implicated to mediate the occurrence of cerebral infarction after SAH, ischemia being one among the many putative pathways. Cerebral infarction due to DCI is now thought to be a better prognostic factor for functional outcome than DCI alone.$^{6,21,24}$ Although a number of studies have identified predictors of DCI and cerebral infarction, our study is most likely one of the largest series. We demonstrated the impact of the primary injury and SAH clot burden in the pathogenesis of DCI and infarct formation after SAH. While the aneurysm size was the more relevant additional predictor for infarct formation, age and sex were more relevant additional predictors of DCI. Indeed, previous studies have identified multiple predictors, including those indicative of the severity of the primary injury and SAH clot burden, modifiable risk factors related to lifestyle habits, or the secondary complications of the aneurysm, including vasospasm, hydrocephalus requiring drainage, and elevated intracranial pressure. In the meta-analysis of de Rooij et al. involving 52 studies that evaluated 33 potential predictors of DCI, the most robust evidence was found for a history of cigarette smoking (pooled OR 1.2, 95\% CI 1.11.4). ${ }^{3}$ The other predictors identified included hyperglycemia, hydrocephalus, diabetes mellitus, and early systemic inflammatory response syndrome. In a recent study of 632 patients who were managed in a single center, predictors of early cerebral infarction were identified and included Hunt and Hess Grades IV and V (OR $=2.06, \mathrm{p}=0.008)$, Fisher Grades 3 and $4(\mathrm{OR}=3.99, \mathrm{p}=0.014)$, sustained elevations of intracranial pressure $>20 \mathrm{~mm} \mathrm{Hg}$ (OR 5.95, p < 0.0001), and early vasospasm on diagnostic angiography (OR 3.01, $\mathrm{p}=0.008) .{ }^{10}$ In a related paper, the authors reported a risk score for cerebral infarction (BEHAVIOR score) based on 7 independent predictors identified at multivariable analysis, including Fisher grade of SAH clot, patient age $\geq 55$ years, Hunt and Hess grade, acute hydrocephalus requiring external CSF drainage, vasospasm on initial angiography, intracranial pressure greater than $20 \mathrm{~mm} \mathrm{Hg}$, and treatment of multiple aneurysms. ${ }^{11}$ In addition, Kanamaru et al. recently published their post hoc analysis of predictors for cerebral infarction in 579 patients in the Prospective Registry of Subarachnoid Aneurysms Treatment (PRESAT). ${ }^{13}$
The predictors were Fisher Grade 3 on admission, larger aneurysm dome size, ruptured posterior circulation aneurysms, premature aneurysm rupture during the clipping procedure, symptomatic vasospasm, and infection.

Because of missing data, we could not examine a number of the important factors identified in previous studies. This is especially true for cigarette smoking, as this was a strong predictor in the study of de Rooij et al. ${ }^{3}$ Moreover, there were variations in the definition of variables, for example, vasospasm, which necessitated the exclusion. The selection of the outcome parameters DCI and delayed cerebral infarction was based on the definitions of the included studies, which could have caused heterogeneity. It is noteworthy that all trials included in the SAHIT data repository were investigating a treatment for DCI or delayed cerebral infarction. Some of these treatments did reduce DCI or delayed cerebral infarction, which could have biased the results of our study. Furthermore, we were not able to retrieve data on hormone replacement therapy, although this might have influenced the risk of DCI. In menopausal women, hormone replacement therapy appears to reduce the risk of aneurysmal SAH but is not associated with improvement in outcome. ${ }^{18}$ Its relationship to DCI is unknown. Finally, the ratio of treatment modalities in our study is in favor of surgical clipping. The current ratio between clipping and coiling is more toward the latter, which is associated with less risk of DCI. ${ }^{4,5,8}$ Theoretically, this increased use of coiling will lead to a lower incidence of DCI in current aneurysmal SAH populations, which might have an impact on the sex differences. Despite these limitations, our study has some strengths worth highlighting. This study is quite likely the largest series to study DCI and cerebral infarction after SAH. We also achieved a good case mix by the inclusion of prospective studies and databases from multiple regions, including Europe, Asia, Australia, and North America, which make the results of this study more generalizable than those of prior studies.

\section{Conclusions}

The present study indicated that female sex is associated with a higher risk of DCI, and thus sex may play a role in the pathogenesis of DCI but was not associated with menopausal status. Other sex-specific factors may be involved, and we could not fully rule out the influence of hormonal changes on DCI and infarct formation after SAH. The study has identified the predictors of DCI and cerebral infarction in a very large cohort reflecting experience from multiple institutions.

\section{Acknowledgments}

We thank Marc Settino and Winnie Qian (both working at the Li Ka Shing Knowledge Institute, St. Michael's Hospital, Toronto, ON, Canada) for their assistance in development of the data collection protocol and data extraction.

\section{Appendix \\ SAHIT Collaborators}

Walter van den Bergh: Department of Intensive Care, Univer- 
sity Medical Center Groningen, the Netherlands; Nima Etminan: Department of Neurosurgery, Medical Faculty Heinrich Heine University, Düsseldorf, Germany; Daniel Hanggi: Department of Neurosurgery, Medical Faculty Heinrich Heine University, Düsseldorf, Germany; David Hasan: The Roy J. and Lucille A. Carver School of Medicine, University of Iowa, Iowa City, Iowa; S. Claiborne Johnston: Dell Medical School, University of Texas, Austin, Texas; Peter Kirkpatrick: Department of Clinical Neurosciences, Addenbrooke's Hospital, University of Cambridge, United Kingdom; Peter Le Roux: MLHC Brain \& Spine Center at Lankenau Medical Center, Wynnewood, Pennsylvania; Stephan Mayer: Institute for Critical Care Medicine, Icahn School of Medicine at Mount Sinai, New York, New York; Andrew Molyneux: The Manor Hospital, Oxford, United Kingdom; Adam Noble: Department of Psychological Sciences, Institute of Psychology, Health \& Society, University of Liverpool, United Kingdom; John Pickard: Department of Clinical Neurosciences, Addenbrooke's Hospital, University of Cambridge, United Kingdom; Audrey Quinn: The General Infirmary, Leeds, United Kingdom; Karl Schaller: Service of Neurosurgery, University Hospital of Geneva, Switzerland; Thomas Schenk: Department of Neurology, Friedrich-Alexander University, Erlangen-Nuremberg, Germany; Tom Schweizer: Keenan Research Centre of the Li Ka Shing Knowledge Institute, St Michael's Hospital, Toronto, Ontario, Canada; Julian Spears: Division of Neurosurgery, St. Michael's Hospital, University of Toronto, Ontario, Canada; Michael Todd: Department of Anesthesia, Carver College of Medicine, University of Iowa, Iowa City, Iowa; James Torner: Department of Epidemiology, College of Public Health, University of Iowa, Iowa City, Iowa; Ming-Yuan Tseng: Medicines and Healthcare Products Regulatory Agency, University of Cambridge, United Kingdom; Mervyn Vergouwen: Department of Neurology, University Medical Center Utrecht, the Netherlands; and George Wong: Department of Surgery, Chinese University of Hong Kong, China.

\section{References}

1. Algra AM, Klijn CJ, Helmerhorst FM, Algra A, Rinkel GJ: Female risk factors for subarachnoid hemorrhage: a systematic review. Neurology 79:1230-1236, 2012

2. Budohoski KP, Guilfoyle M, Helmy A, Huuskonen T, Czosnyka M, Kirollos R, et al: The pathophysiology and treatment of delayed cerebral ischaemia following subarachnoid haemorrhage. J Neurol Neurosurg Psychiatry 85:1343-1353, 2014

3. de Rooij NK, Rinkel GJ, Dankbaar JW, Frijns CJ: Delayed cerebral ischemia after subarachnoid hemorrhage: a systematic review of clinical, laboratory, and radiological predictors. Stroke 44:43-54, 2013

4. Dorhout Mees SM, Kerr RS, Rinkel GJ, Algra A, Molyneux $\mathrm{AJ}$ : Occurrence and impact of delayed cerebral ischemia after coiling and after clipping in the International Subarachnoid Aneurysm Trial (ISAT). J Neurol 259:679-683, 2012

5. Dumont AS, Crowley RW, Monteith SJ, Ilodigwe D, Kassell $\mathrm{NF}$, Mayer S, et al: Endovascular treatment or neurosurgical clipping of ruptured intracranial aneurysms: effect on angiographic vasospasm, delayed ischemic neurological deficit, cerebral infarction, and clinical outcome. Stroke 41:25192524, 2010

6. Etminan N, Vergouwen MD, Macdonald RL: Angiographic vasospasm versus cerebral infarction as outcome measures after aneurysmal subarachnoid hemorrhage. Acta Neurochir Suppl 115:33-40, 2013

7. Fisher CM, Kistler JP, Davis JM: Relation of cerebral vasospasm to subarachnoid hemorrhage visualized by computerized tomographic scanning. Neurosurgery 6:1-9, 1980

8. Germans MR, Macdonald RL: Clip or coil-is some of the effect on outcome related to the risk of delayed cerebral ischemia? World Neurosurg 82:e679-e681, 2014

9. Horikoshi T, Akiyama I, Yamagata Z, Sugita M, Nukui H:
Magnetic resonance angiographic evidence of sex-linked variations in the circle of Willis and the occurrence of cerebral aneurysms. J Neurosurg 96:697-703, 2002

10. Jabbarli R, Reinhard M, Niesen WD, Roelz R, Shah M, Kaier $\mathrm{K}$, et al: Predictors and impact of early cerebral infarction after aneurysmal subarachnoid hemorrhage. Eur J Neurol 22:941-947, 2015

11. Jabbarli R, Reinhard M, Roelz R, Shah M, Niesen WD, Kaier $\mathrm{K}$, et al: Early identification of individuals at high risk for cerebral infarction after aneurysmal subarachnoid hemorrhage: the BEHAVIOR score. J Cereb Blood Flow Metab 35:1587-1592, 2015

12. Jaja BN, Attalla D, Macdonald RL, Schweizer TA, Cusimano MD, Etminan N, et al: The Subarachnoid Hemorrhage International Trialists (SAHIT) Repository: advancing clinical research in subarachnoid hemorrhage. Neurocrit Care 21:551-559, 2014

13. Kanamaru K, Suzuki H, Taki W: Cerebral infarction after aneurysmal subarachnoid hemorrhage. Acta Neurochir Suppl 121:167-172, 2016

14. Li H, Pan R, Wang H, Rong X, Yin Z, Milgrom DP, et al: Clipping versus coiling for ruptured intracranial aneurysms: a systematic review and meta-analysis. Stroke 44:29-37, 2013

15. Lindekleiv H, Sandvei MS, Njølstad I, Løchen ML, Romundstad PR, Vatten L, et al: Sex differences in risk factors for aneurysmal subarachnoid hemorrhage: a cohort study. Neurology 76:637-643, 2011

16. Macdonald RL: Delayed neurological deterioration after subarachnoid haemorrhage. Nat Rev Neurol 10:44-58, 2014

17. Macdonald RL, Cusimano MD, Etminan N, Hanggi D, Hasan D, Ilodigwe D, et al: Subarachnoid Hemorrhage International Trialists data repository (SAHIT). World Neurosurg 79:418-422, 2013

18. Mhurchu CN, Anderson C, Jamrozik K, Hankey G, Dunbabin D: Hormonal factors and risk of aneurysmal subarachnoid hemorrhage: an international population-based, case-control study. Stroke 32:606-612, 2001

19. Phipps AI, Ichikawa L, Bowles EJ, Carney PA, Kerlikowske $\mathrm{K}$, Miglioretti DL, et al: Defining menopausal status in epidemiologic studies: A comparison of multiple approaches and their effects on breast cancer rates. Maturitas 67:60-66, 2010

20. Reilly C, Amidei C, Tolentino J, Jahromi BS, Macdonald RL: Clot volume and clearance rate as independent predictors of vasospasm after aneurysmal subarachnoid hemorrhage. J Neurosurg 101:255-261, 2004

21. Rosengart AJ, Schultheiss KE, Tolentino J, Macdonald RL: Prognostic factors for outcome in patients with aneurysmal subarachnoid hemorrhage. Stroke 38:2315-2321, 2007

22. Tabuchi S: Relationship between postmenopausal estrogen deficiency and aneurysmal subarachnoid hemorrhage. Behav Neurol 2015:720141, 2015

23. Teasdale GM, Drake CG, Hunt W, Kassell N, Sano K, Pertuiset B, et al: A universal subarachnoid hemorrhage scale: report of a committee of the World Federation of Neurosurgical Societies. J Neurol Neurosurg Psychiatry 51:1457, 1988

24. Vergouwen MD: Vasospasm versus delayed cerebral ischemia as an outcome event in clinical trials and observational studies. Neurocrit Care 15:308-311, 2011

25. Wilson DA, Nakaji P, Abla AA, Uschold TD, Fusco DJ, Oppenlander ME, et al: A simple and quantitative method to predict symptomatic vasospasm after subarachnoid hemorrhage based on computed tomography: beyond the Fisher scale. Neurosurgery 71:869-875, 2012

\section{Disclosures}

Dr. Macdonald states that he is Chief Scientific Officer of Edge 
Therapeutics Inc., is a consultant for Actelion Pharmaceuticals, Ltd., has direct stock ownership in Edge Therapeutics, receives non-study-related clinical/research support, and has ownership in the Heart and Stroke Foundation of Canada and the Brain Aneurysm Foundation.

\section{Author Contributions}

Conception and design: Germans, de Oliveira Manoel, Macdonald. Acquisition of data: Germans, Cohen. Analysis and interpretation of data: Germans, Jaja, Cohen. Drafting the article: Germans, Jaja, de Oliveira Manoel. Critically revising the article: all authors. Reviewed submitted version of manuscript: Germans, Jaja, Macdonald. Approved the final version of the manuscript on behalf of all authors: Germans. Statistical analysis: Jaja. Study supervision: Macdonald.

\section{Supplemental Information}

Previous Presentations

Portions of this work were presented in poster form at the EANS2016 Congress, September 4-8, 2016, Athens, Greece.

Online-Only Content

Supplemental material is available with the online version of the article.

Supplemental Table. https://thejns.org/doi/suppl/10.3171/2017. 3.JNS162808.

\section{Correspondence}

Menno R. Germans, Department of Neurosurgery, Radboud University Medical Center, PO Box 9101, Nijmegen 6500 HB, The Netherlands.email: mrgermans@hotmail.com. 